\title{
MicroRNA expression profiles of granulocytic myeloid-derived suppressor cells from mice bearing Lewis lung carcinoma
}

\author{
JINGWEI JIANG ${ }^{1,2^{*}}$, QINGMIN GAO ${ }^{1,2^{*}}$, TIAN WANG ${ }^{1,2}$, HAO LIN $^{1,2}$, QIONG ZHAN $^{1,2}$, ZHAOHUI CHU $^{1,2}$ \\ RUOFAN HUANG ${ }^{1,2}$, XINLI ZHOU ${ }^{1,2}$, XIAOHUA LIANG ${ }^{1,2}$ and WEIJIAN GUO ${ }^{2,3}$ \\ ${ }^{1}$ Department of Oncology, Huashan Hospital, Fudan University, Shanghai 200040; ${ }^{2}$ Department of Oncology, \\ Shanghai Medical College; ${ }^{3}$ Department of Medical Oncology, Fudan University Shanghai Cancer Center, \\ Fudan University, Shanghai 200032, P.R. China
}

Received September 8, 2015; Accepted September 22, 2016

DOI: $10.3892 / \mathrm{mmr} .2016 .5845$

\begin{abstract}
Myeloid-derived suppressor cells (MDSCs) are a group of heterogeneous myeloid cells that can suppress antitumor immunity. MDSCs are divided into granulocytic (G-MDSCs) and monocytic subsets. In the present study, the microRNA profiles of the G-MDSCs were determined and the differential expression of microRNAs between G-MDSCs from tumor-bearing mice and tumor-free mice was examined. The number of G-MDSCs in spleens of Lewis lung carcinoma (LLC)-bearing mice was $\sim 6$-fold higher than in spleens of normal mice $(13.54 \pm 1.74 \%$ vs. $2.14 \pm 1.44 \%$; $\mathrm{P}<0.01)$ and G-MDSCs account for about $72.9 \%$ of all MDSCs. The microRNA (miRNA) profiles of the G-MDSCs from spleen of LLC-bearing mice were obtained using a microRNA microarray and compared with their counterparts from spleens of tumor-free mice. A total of 43 miRNAs with $>1$.3-fold increased or decreased change were differentially expressed between the experimental and control group mice. The levels of nine of these differentially expressed miRNAs, miRNA-468 (miR-486), miR-192, miR-128, miR-125a, miR-149, miR-27a, miR-125b, miR-350 and miR-328, were also analyzed by RT-qPCR to validate the microarray data. The concordance rate between the results tested by the two methods was $88.9 \%$. Bioinformatics analyses revealed that these miRNAs may act on various target
\end{abstract}

Correspondence to: Dr Weijian Guo, Department of Medical Oncology, Fudan University Shanghai Cancer Center, Fudan University, 270 Dong'an Road, Xuhui, Shanghai 200032, P.R. China E-mail: guoweijiansh@163.com

Abbreviations: MDSCs, myeloid-derived suppressor cells; LLC, Lewis lung carcinoma; G-MDSCs, granulocytic myeloid-derived suppressor cells; M-MDSCs, monocytic MDSCs; miRNAs, microRNAs; RT-qPCR, reverse transcription-quantitative polymerase chain reaction

*Contributed equally

Key words: myeloid-derived suppressor cells, microRNA, immunoregulation, tumor immune, bioinformatics analysis genes, including Adar, Pik3r1, Rybp and Rabgap1, to regulate the survival, differentiation and the function of tumor-induced granulocytic MDSCs. The results revealed microRNAs and potential targets that may be vital for regulating survival, differentiation and function of G-MDSCs induced by LLC. Further investigation should be performed to clarify the roles of these microRNAs in regulating LLC-induced granulocytic MDSCs and the target genes that mediate their functions.

\section{Introduction}

Myeloid-derived suppressor cells (MDSCs) are a group of heterogeneous cells originating from myeloid cells and they are at different stages of differentiation. MDSCs expand during infection and inflammation, and particularly during cancer. MDSCs have a strong ability to suppress anti-tumor immunity, such as T-cell responses. The proportion of MDSCs was demonstrated to be $20-40 \%$ of nucleated splenocytes in several mouse tumor models, whereas the proportion of MDSCs in spleens of tumor-free mice was 2-4\% $(1,2)$. In a mouse model, MDSCs are typically identified through expression of lymphocyte antigen 6 complex, locus G (Ly6G) and CD11b, which are myeloid lineage differentiation markers (1-3). It is now accepted that MDSCs can be divided into two major subsets; polymorphonuclear and mononuclear morphology, which are distinguished by a combination of specific markers. Polymorphonuclear or granulocytic MDSCs (G-MDSCs) are positive for CD11b and Ly6G, and exhibit low expression of Ly6C, whereas mononuclear or monocytic MDSCs (M-MDSCs) are positive for CD11b, negative for Ly6G and exhibit high expression of Ly6C (1-3). G-MDSCs are the largest subset of MDSCs in tumor-bearing mice, which account for $>80 \%$ of all MDSCs (1-3). Previous reports have suggested that G-MDSCs and M-MDSCs subsets may suppress antigen-specific immune responses through different signaling pathways and mechanisms. M-MDSCs were demonstrated to suppress antigen-specific responses via nitric oxide, which was dependent on interferon (IFN)- $\gamma$ (4). However, G-MDSCs produced higher levels of reactive oxygen species (ROS), and their inhibitory function was completely reversed by ROS scavengers $(3,4)$. The production and functional regulation mechanisms of G-MDSCs and M-MDSCs may also be different. For example, interleukin-18 specifically 
enhanced the differentiation and function of M-MDSCs, but not G-MDSCs (5). IFN- $\gamma$ was reported to control the survival and function of tumor-induced G-MDSCs by suppressing the mRNA expression levels of the anti-apoptotic molecule B cell leukemia/lymphoma 2 related protein Ala (6).

MicroRNAs (miRNAs) are small, single-stranded non-coding RNAs. Many are highly conserved (7). Gene expression regulation by miRNAs affects various biological processes, including cell differentiation, cell proliferation, cell apoptosis, signal transduction, immune responses and hematopoiesis. MiRNAs predominantly decrease the expression of their target genes through inducing degradation of mRNA or inhibiting mRNA translation, however there is also evidence supporting that miRNAs may increase the expression of certain target genes (8). MDSCs that originate from hematopoietic stem cells are highly accumulated in the spleen, peripheral blood, lymphoid organs and tumor tissue in tumor-bearing mice and patients with cancer. MDSCs are essentially undifferentiated hematopoietic cells and are also immune-regulating cells (9). Therefore, the production and function of MDSCs may be regulated by miRNAs. Recent studies have authenticated this assumption. For example, miRNA (miR)-17 and miR-20a attenuate the suppressive potential of MDSCs through modulating signal transducer and activator of transcription (STAT) 3 expression (10). MiR-494 is required for the accumulation and function of tumor-expanded MDSCs by targeting the mRNA of phosphatase and tensin homolog (PTEN) (11). MiR-155 and miR-21 promote the expansion of functional MDSCs (12). Twist basic helix-loop-helix transcription factor and miR-34a participate in the generation of tumor-induced MDSCs (13). MiR-34a expands MDSCs via apoptosis inhibition (14). Determining the miRNA profiles of MDSCs may provide novel insight into the molecular mechanisms and illuminate the features of tumor-induced MDSCs, which will facilitate the design and development of novel anti-cancer drugs. MiRNA microarrays have been performed by Liu et al (11) and Li et al (12) to analyze the miRNA profiles of tumor-induced MDSCs, however, the miRNA microarrays were conducted using the whole MDSC population, not MDSCs subtypes $(11,12)$. As the G-MDSCs and M-MDSCs have different mechanisms of production and function, the miRNA profiles of these two subsets may also be different.

The current study aimed to identify the miRNA profiles of G-MDSCs induced by malignant tumor using a GeneChip miRNA 3.0 array, and to predict the function of the miRNAs in tumor-induced G-MDSCs using bioinformatics analysis. C57BL/6 mice bearing Lewis lung carcinoma (LLC) is a widely used model for preclinical studies of MDSCs. To address the scientific objectives of the present study, G-MDSCs from spleens of mice bearing LLC were used as the experimental group and G-MDSCs from spleens of tumor-free mice were used as the control group.

\section{Materials and methods}

Cell line. LLC cell line was purchased from the Cell Resource Center of Shanghai Institutes for Biological Sciences, Chinese Academy of Sciences (Shanghai, China). LLC tumor cells were maintained in Dulbecco's modified Eagle's medium (Hyclone; GE Healthcare Life Sciences, Logan, UT, USA) supplemented with $10 \%$ fetal bovine serum (Hyclone; GE Healthcare Life Sciences) and $1 \%$ penicillin-streptomycin (Gibco; Thermo Fisher Scientific, Inc., Waltham, MA, USA). Cells were maintained at $37^{\circ} \mathrm{C}$ in a humidified atmosphere containing $95 \%$ air and $5 \% \mathrm{CO}_{2}$.

Animal models. A total of 32 female C57BL/6 mice at 4-6 weeks of age were provided by the Department of Laboratory Animal Science of Fudan University (Shanghai, China) and were housed under specific pathogen-free conditions. Groups of three to five mice were housed in polypropylene cages with sterilized bedding under controlled conditions: Temperature, $24 \pm 1^{\circ} \mathrm{C}$; relative humidity, 55\%. The mice had ad libitum access to a standard diet and sterilized water; water bottles were replaced daily. All animal experiments were performed in accordance with approval protocol for animal use and handling. Mice $(n=12)$ were subcutaneously injected in the flank with $1 \times 10^{6}$ LLC cells and were sacrificed for further analysis when the tumor reached 15-20 mm diameter within 3-4 weeks after injection. Tumor-free mice $(n=20)$ were considered the control group, and were sacrificed at 7-9 weeks old. Prior to sacrifice, mice were anesthetized by $2 \%$ sodium pentobarbital (Sigma-Aldrich; Merck Millipore, Darmstadt, Germany); the mice were then sacrificed by cervical dislocation and spleens were collected. The study was approved by the Animal Welfare and Ethics Committee of the Department of Laboratory Animal Science, Fudan University (permit no. 20150295A010).

G-MDSC purification. Mice spleens were dissociated mechanically and individual spleen cells were obtained through a $75-\mu \mathrm{m}$ cellular sieve, and then centrifuged at $250 \mathrm{x} g$ for $5 \mathrm{~min}$ at $4^{\circ} \mathrm{C}$. Single-cell suspensions were passed through a $30-\mu \mathrm{m}$ nylon mesh (pre-separation filters; cat. no. 130-041-407; Miltenyi Biotec GmbH, Bergisch Gladbach, Germany). Red blood cells were eliminated using ACK Lysis Buffer (Leagene Biotech Co., Ltd., Beijing, China) in accordance with the manufacturer's instructions. Cells were counted and washed with PBS. Single-cell suspensions $\left(1 \times 10^{6} / \mathrm{L}\right.$ per sample) prepared from the spleen were stained with the following specific fluorophore-conjugated anti-mouse antibodies in PBS for $15 \mathrm{~min}$ in the dark: CD11b-allophycocyanin $(1.25 \mu \mathrm{g} / \mathrm{ml}$; cat. no. M1/70; eBioscience, Inc., San Diego, CA, USA), Ly6G-phycoerythrin (18.2 $\mu \mathrm{g} / \mathrm{ml}$; cat. no. 1A8; BD Biosciences, Franklin Lakes, NJ, USA), and Ly6C-PerCP-Cy5.5 (9.5 $\mu \mathrm{g} / \mathrm{ml}$; cat. no. HK1.4; eBioscience, Inc.). The binding specificity of each antibody was confirmed using its corresponding isotype. The percentages of MDSCs and MDSC subsets were determined using a BD FACSCanto ${ }^{\mathrm{TM}}$ flow cytometer (BD Biosciences), and G-MDSCs were purified using a MoFlo flow cytometer (Dakocytomation; Agilent Technologies, Inc., Santa Clara, CA, USA). For purification of G-MDSCs, CD11b-positive cells were gated. The G-MDSC subset was specifically sorted by Ly6G and Ly6C detection. The purity of G-MDSCs was $>90 \%$. Fluorescence data were analyzed using FlowJo version 5.7.2 software (Tree Star, Inc., Ashland, OR, USA). Each sample used G-MDSCs from spleens of three mice bearing LLC tumors as the experimental group or from the spleens of five tumor-free mice as the control group. 
RNA extraction and miRNA array assay. The isolated G-MDSCs were maintained in TRIzol LS (Invitrogen; Thermo Fisher Scientific, Inc.). Total RNA was extracted and purified using the miRNeasy Mini kit (Qiagen $\mathrm{GmbH}$, Hilden, Germany) and RNase-free DNase 1 (Qiagen $\mathrm{GmbH}$ ) according to the manufacturer's instructions. The concentration of RNA was measured by an SMA3000 spectrophotometer (Merinton Instrument, Ltd., Beijing, China). Optical density 260/280 value between 1.9 and 2.1 was considered as good purity. The integrity and quality of RNA was evaluated by JS-380A Gel Imaging Analysis System (Peiqing Science And Technology Co., Ltd., Shanghai, China).

Qualified RNA samples were further analyzed using the GeneChip miRNA 3.0 array (Affymetrix, Inc., Santa Clara, CA, USA) at GMINIX Information Technology, Ltd. (Shanghai, China). This array is a single array comprised of 179,217 probes that represent 19,913 mature miRNAs contained in the miRBase V.17 (www.mirbase.org). RNA was labeled with biotin and hybridized with the array according to the manufacturer's instructions. Signal scanning was conducted by a GeneChip Scanner (Affymetrix, Inc.). Analysis of each sample was repeated twice. The mean was used for comparisons.

Reverse transcription-quantitative polymerase chain reaction (RT-qPCR) analysis. Total RNA was purified from G-MDSCs of LLC-bearing and control mice using TRIzol ${ }^{\circledR}$ (Invitrogen; Thermo Fisher Scientific, Inc.) in accordance with the manufacturer's protocols. The concentration of RNA was determined by a NanoDrop 2000 spectrophotometer (Thermo Fisher Scientific, Inc., Pittsburgh, PA, USA). RNA integrity was evaluated using agarose gel electrophoresis and ethidium bromide staining. cDNA was synthesized from $1 \mu \mathrm{g}$ total RNA using miScript II Reverse Transcriptase Mix (Qiagen $\mathrm{GMbH}$ ) according to the manufacturer's instructions. Reactions were performed using a GeneAmp ${ }^{\circledR}$ PCR system 9700 (Applied Biosystems; Thermo Fisher Scientific, Inc.) for $60 \mathrm{~min}$ at $37^{\circ} \mathrm{C}$, followed by heat inactivation of RT for $5 \mathrm{~min}$ at $95^{\circ} \mathrm{C}$. The $20 \mu \mathrm{l}$ RT reaction mixture was then diluted $5 \mathrm{X}$ in nuclease-free water and maintained at $-20^{\circ} \mathrm{C}$. RT-qPCR was conducted with SYBR Green I Master kit (Roche Diagnostics, Basel, Switzerland) in accordance with the manufacturer's protocol using a Light Cycler 480 II RT-PCR platform (Roche Diagnostics). The $10 \mu \mathrm{l}$ PCR reaction mixture consisted of $1 \mu \mathrm{l}$ cDNA, $5 \mu \mathrm{l}$ 2X SYBR Green I Master, $0.2 \mu \mathrm{l}$ universal reverse primer (Qiagen $\mathrm{GmbH}$ ), $0.2 \mu 1$ miRNA-specific primer and $3.6 \mu 1$ nuclease-free water. Reactions were incubated at $95^{\circ} \mathrm{C}$ for $10 \mathrm{~min}$, followed by 40 cycles at $95^{\circ} \mathrm{C}$ for $10 \mathrm{sec}$ and $60^{\circ} \mathrm{C}$ for $30 \mathrm{sec}$. Each sample was run in triplicate for analysis. At the end of the PCR, a melting curve analysis was performed to validate the specific generation of the expected PCR product. 5S small RNA was used as the endogenous control to normalize the expression of miRNAs. The miRNAs analyzed by RT-qPCR included: MiR-486, miR-192, miR-128, miR-125a, miR-149, miR-27a, miR-125b, miR-350 and miR-328. Primer sequences used for these miRNAs are listed in Table I. The experiments were repeated three times independently. The comparative $2^{-\Delta \Delta C q}$ method was used to calculate the relative expression level of miRNAs (15).

Target prediction and bioinformatics analyses. Four online software programs, MirTarget2 (mirdb.org/miRDB), PicTar (pictar.mdc-berlin.de), miRanda (microrna.sanger.ac.uk), and
Table I. Primers used for quantitative polymerase chain reaction.
$\operatorname{miR}$
Primer sequence $\left(5^{\prime}>3^{\prime}\right)$

$5 \mathrm{~S}$

mmu-miR-486-5p

mmu-miR-192-5p

mmu-miR-99b-5p

mmu-miR-128-3p

GGAGACCGCCTGGGAATA

mmu-miR-125a-5p

mmu-miR-149-5p

TCCTGTACTGAGCTGCCCCGAG

CTGACCTATGAATTGACAGCC

CACCCGTAGAACCGACCTTGCG

TCACAGTGAACCGGTCTCTTT

TCCCTGAGACCCTTTAACCTGTGA

mmu-miR-27a-5p

TCTGGCTCCGTGTCTTCACTCCC

mmu-miR-125b-5p

AGGGCTTAGCTGCTTGTGAGCA

mmu-miR-350-3p

TCCCTGAGACCCTAACTTGTGA

mmu-miR-328-3p

TTCACAAAGCCCATACACTTTC

CTGGCCCTCTCTGCCCTTCCGT

PITA (genie.weizmann.ac.il/pubs/mir07/mir07_prediction. html) were used to search for the mouse target genes of the nine RT-qPCR validated miRNAs. Probability distribution of random matches was set at 0.05 , which was Poisson P-value. Targets with $\mathrm{P}<0.05$ and predicted by all of the four algorithms were regarded as predicted targets. The functional enrichment and pathway analyses of the predicted target genes were performed using the Gene Ontology (GO; geneontology.org) and Kyoto Encyclopedia of Genes and Genomes (KEGG; www.kegg.jp) online databases. Maps of miRNAs and their target genes were created using Cytoscape (www.cytoscape.org). This is an open source of bioinformatics platform for analyzing and visualizing complex networks (16), which are equipped with ClueGo and CluePedia plugins (17).

Statistical analysis. Stata/SE 10.1 software (StataCorp LP, College Station, TX, USA) was used to analyze the data. Data are presented as the mean \pm standard deviation. Statistical analyses were conducted using a two-tailed Student's $t$-test. For miRNA array assay, both $>1.3$-fold increased or decreased change and $\mathrm{P}<0.05$ were considered to indicate a statistically significant difference. For all other tests; $\mathrm{P}<0.05$ was considered to indicate a statistically significant difference.

\section{Results}

Accumulation of G-MDSCs in the spleens of mice with LLC. Nucleated spleen cells were obtained from mice bearing LLC and normal mice, and the percentages of CD11b-positive cells, G-MDSCs and M-MDSCs subsets were detected by flow cytometry (Fig. 1A and B). The percentage of CD11b-positive cells was increased in spleens of mice bearing LLC compared with normal mice $(18.58 \pm 2.70 \%$ vs. $3.08 \pm 1.97 \%$; $\mathrm{P}<0.01$; Fig. 1C); After CD11b positive cells were gated, both the CD11b ${ }^{+}$Ly6G ${ }^{+}$Ly6C $^{\text {low }}$ G-MDSCs and CD11b ${ }^{+}$Ly6G-Ly6C ${ }^{\text {high }}$ M-MDSCs were demonstrated to be increased in the spleens of mice bearing LLC compared with normal mice. The percentages of MDSCs, G-MDSCs and M-MDSCs in spleens of mice bearing LLC were $\sim 6$-fold higher that their counterparts from 

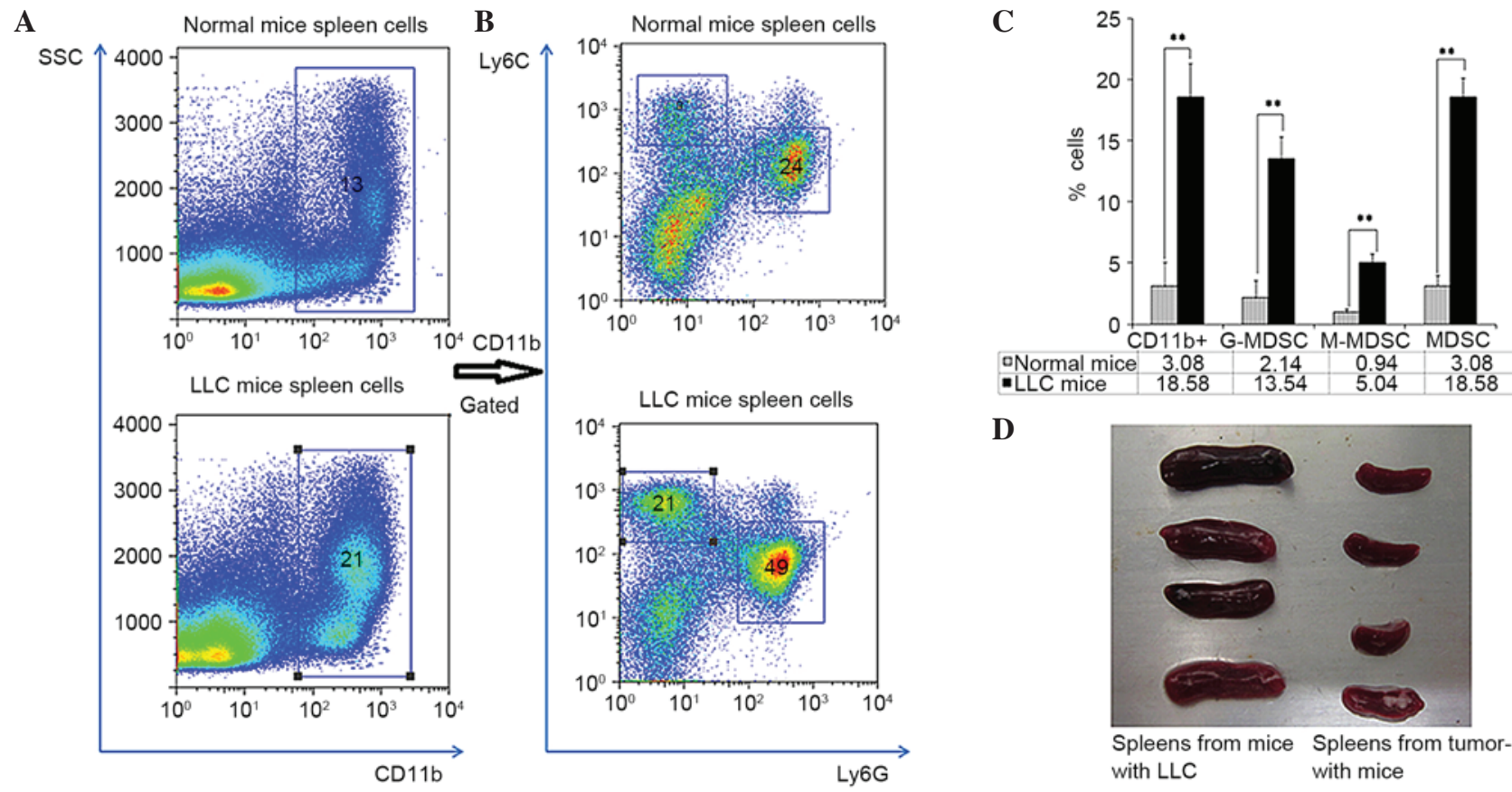

Figure 1. Accumulation of G-MDSCs in the spleen of mice with Lewis lung carcinoma. (A) The percentage of CD11b positive cells increased in spleens of mice bearing LLC compared to that of normal mice. (B) After CD11b positive cells were gated, both the G-MDSCs (CD11b $\left.{ }^{+} \mathrm{Ly} 6 \mathrm{G}^{+} \mathrm{Ly} 6 \mathrm{C}^{\mathrm{low}}\right)$ and $\mathrm{M}-\mathrm{MDSCs}$ $\left(\mathrm{CD} 11 \mathrm{~b}^{+}\right.$Ly6G-Ly6Chi) were increased in spleens of mice bearing LLC compared with normal mice. (C) The percentages of CD11b ${ }^{+}$cells, MDSCs, G-MDSCs and M-MDSCs in spleens of mice bearing LLC were $\sim 6$-folds higher of those of normal mice. Data are presented as the mean \pm standard deviation. ${ }^{* *} \mathrm{P}<0.01$. (D) The volume of spleens from mice bearing LLC was larger than from tumor-free mice. SSC, side scatter; LLC, Lewis lung carcinoma; Ly6G, lymphocyte antigen 6 complex, locus G; MSDC, myeloid-derived suppressor cells; G-, granulocytic; M-, monocytic.

tumor-free mice $(18.58 \pm 1.56 \%$ vs. $3.08 \pm 0.88 \%$ for MDSCs; $13.54 \pm 1.74 \%$ vs. $2.14 \pm 1.44 \%$ for G-MDSCs; and $5.04 \pm 0.71 \%$ vs. $0.94 \pm 0.32 \%$ for M-MDSCs; all $\mathrm{P}<0.01)$. G-MDSCs account for $72.9 \%$ of all MDSCs. Furthermore, the volume of spleens from mice bearing LLC was larger than from tumor-free mice (Fig. 1D).

MiRNA expression profiles of G-MDSCs from spleens of mice with LLC compared with tumor-free mice. Microarrays were used to evaluate the miRNA expression profiles of the G-MDSCs from spleen of mice with LLC and compare them with the profile in tumor-free mice. The miRNA expression patterns between the two MDSC groups were different. In total, 43 miRNAs that exhibited an increase or decrease of $>1.3$-fold were considered differentially expressed between the G-MDSCs from mice with LLC and tumor-free mice (Fig. 2). Of these miRNAs, 20 were upregulated and 23 miRNAs were downregulated in the G-MDSCs from mice with LLC compared with tumor-free mice.

Validation of results from miRNA array by $R T$-qPCR. RT-qPCR was performed for 9 of the differentially expressed miRNAs (fold change $>2$ ) to validate the results of the miRNA microarray. The relative ratio of miRNA expression between the G-MDSCs from mice bearing LLC with tumor-free mice was determined by RT-qPCR. The relative ratios for the 9 detected miRNAs were 0.6, 2.7, 7.6, 3.3, 1.8, 1.9, 1.4, 4.8 and 3.1 for miR-486, miR-192, miR-128, miR-125a, miR-149, miR-27a, miR-125b, miR-350 and miR-328, respectively. The RT-qPCR data from 8 out of the 9 miRNAs was in accordance with the microarray results (Fig. 3), excluding miR-486. The concordance rate of the results analyzed by the two methods was $88.9 \%$.

Target prediction and functional analyses of the differentially expressed miRNAs. The targets of the $9 \mathrm{miRNAs}$ were predicted using four online software programs: MirTarget2, PicTar, miRanda, and PITA. In order to increase the specificity, the results of the four target prediction programs were integrated and only the genes that were predicted by all the four software programs were analyzed. A total of 729 miRNA-target RNA pairs were identified by all of the four online software programs. For the 9 miRNAs tested by RT-qPCR, 22, 18, 163, 82, 52, 44, 85,228 and 21 targets were predicted for miR-486, miR-192, miR-128, miR-125a, miR-149, miR-27a, miR-125b, miR-350 and miR-328, respectively.

The miRNA-target mRNA pairs were ranked. A network was generated by Cytoscape to demonstrated the association between the predicted miRNA-target gene pairs (Fig. 4). Certain mRNAs were predicted to be potential targets of more than two of these miRNAs. For instance, adenosine deaminase, RNA-specific (Adar) may be a target gene of miR-350, miR-149, miR-128 and miR27a. c-Abl oncogene 1, non-receptor tyrosine kinase (Abl1) may be a target gene of miR-27a, miR-149 and miR-128. Caspase 2 (casp2) may be a target gene of miR149, miR-125a and miR-125b. H3 histone, family 3B (H3f3b) may be a target gene of miR-128, miR-350, miR-21-5p and miR-486. SMEK homolog 1, suppressor of mek1 (Smek1), ELOVL family member 6, elongation of long chain fatty acids (Elovl6) and protogenin (Prtg) may be target genes of miR350, miR-125a and miR-125b. The majority of predicted targets for miR-125a were shared with miR-125b. 


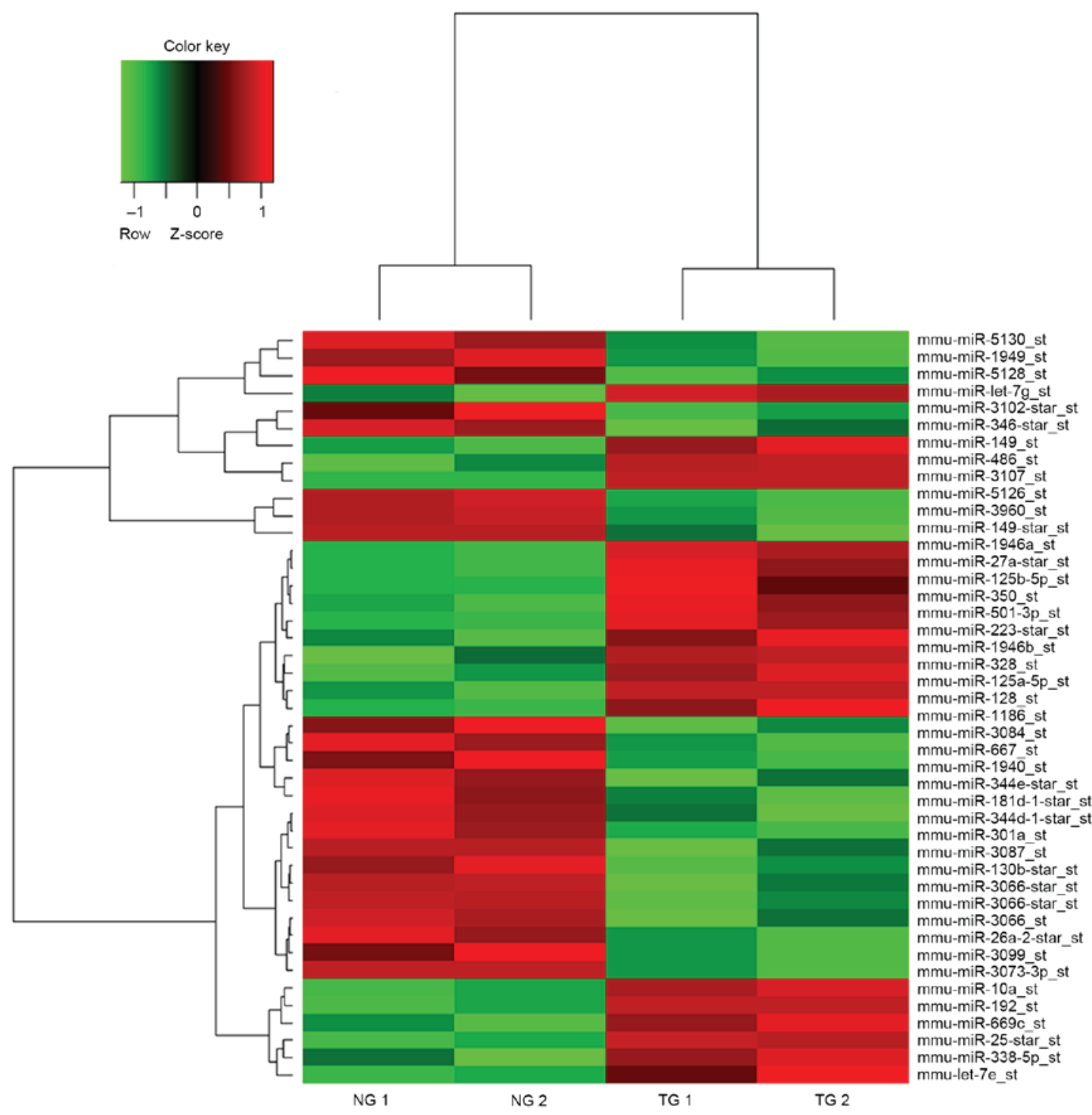

Figure 2. MiRNA expression profiles of LLC-induced G-MDSCs. G-MDSCs from spleens of LLC-bearing mice (TG) and normal mice (NG) were isolated by flow cytometry, then the miRNA expression profiles were analyzed using Affymetrix GeneChip miRNA 3.0 array. The heat map diagram presents the results of the two-way hierarchical clustering of genes and samples, and the clustering was performed on the differentially expressed miRNAs (only differentially expressed miRNAs with fold changes $\geq 1.30$ and $\mathrm{P}<0.05$ are included). The color scale illustrates the relative expression level of an miRNA: Red represents high expression level; green represents low expression level. Each sample column represents data from total RNA pooled from one independent cell sort using three to five mice. miRNA, microRNA.

Predicted target genes were analyzed by GO (Fig. 5A) and KEGG pathway (Fig. 5B) analyses for significant enrichment of genes into functional annotation categories. Significantly enriched categories including cell differentiation, proliferation, apoptotic process, immune response and hematopoiesis are demonstrated with -log (P-value) and the number of target genes. The majority of these targets were involved in regulating signal transducer activity, apoptotic process, cell differentiation, cell cycle and adaptive immune responses (Fig. 5A). Certain target genes associated with several vital signaling pathways of the 'immune cell genes', for example transforming growth factor- $\beta$ (TGF- $\beta$ ), mitogen-activated protein kinase (MAPK), ErbB, Ras, $\mathrm{T}$ cell receptor, tumor necrosis factor (TNF), Wnt and vascular endothelial growth factor (VEGF) signaling pathways (Fig. 5B).

Another concise map of miRNA-target interactions was generated limited to genes that have major functions in regulation of signal transducer activity, apoptotic process, cell differentiation, cell cycle and adaptive immune response (Fig. 6) . Phosphatidylinositol 3-kinase, regulatory subunit, polypeptide 1 (Pik3r1; regulated by miR-486 and miR-128), RING1 and YY1 binding protein (Rybp; regulated by miR-128 and miR-350) and RAB GTPase activating protein 1 (Rabgap1; regulated by miR-27a and miR-192) may also be involved in the regulation of apoptotic process, cell differentiation, cell cycle and adaptive immune response of G-MDSCs.

\section{Discussion}

MDSCs are a heterogeneous group of immature and mature myeloid cells with immunosuppressive activity. MDSCs have been divided into two main subsets; G-MDSCs and M-MDSCs, which have different phenotypic and biological properties. In the majority of tumor models and in many patients with cancer, G-MDSCs are the predominant subgroup, which represent 


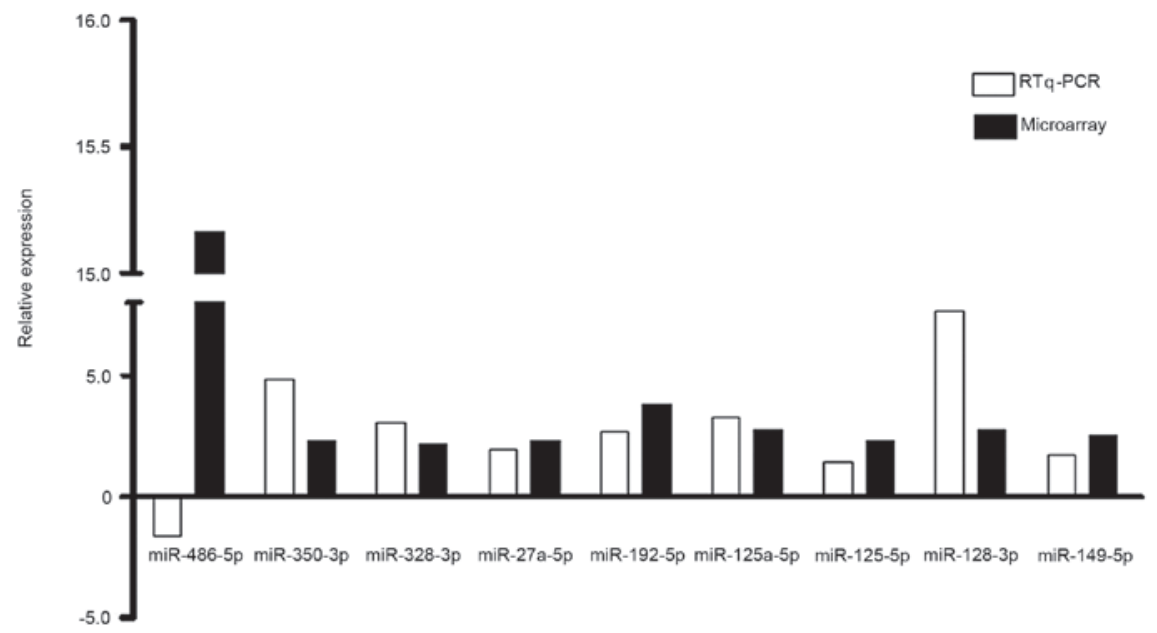

Figure 3. Ratio of experimental to control mice expression levels. Expression of nine miRNAs were approved by RT-qPCR. Eight of the nine tested miRNAs provided RT-qPCR data that were in accordance with the microarray results, not including miR-486. The concordance rate of the results tested by the two methods was $88.9 \%$. RT-qPCR, reverse transcription-quantitative polymerase chain reaction; miRNA, microRNA.

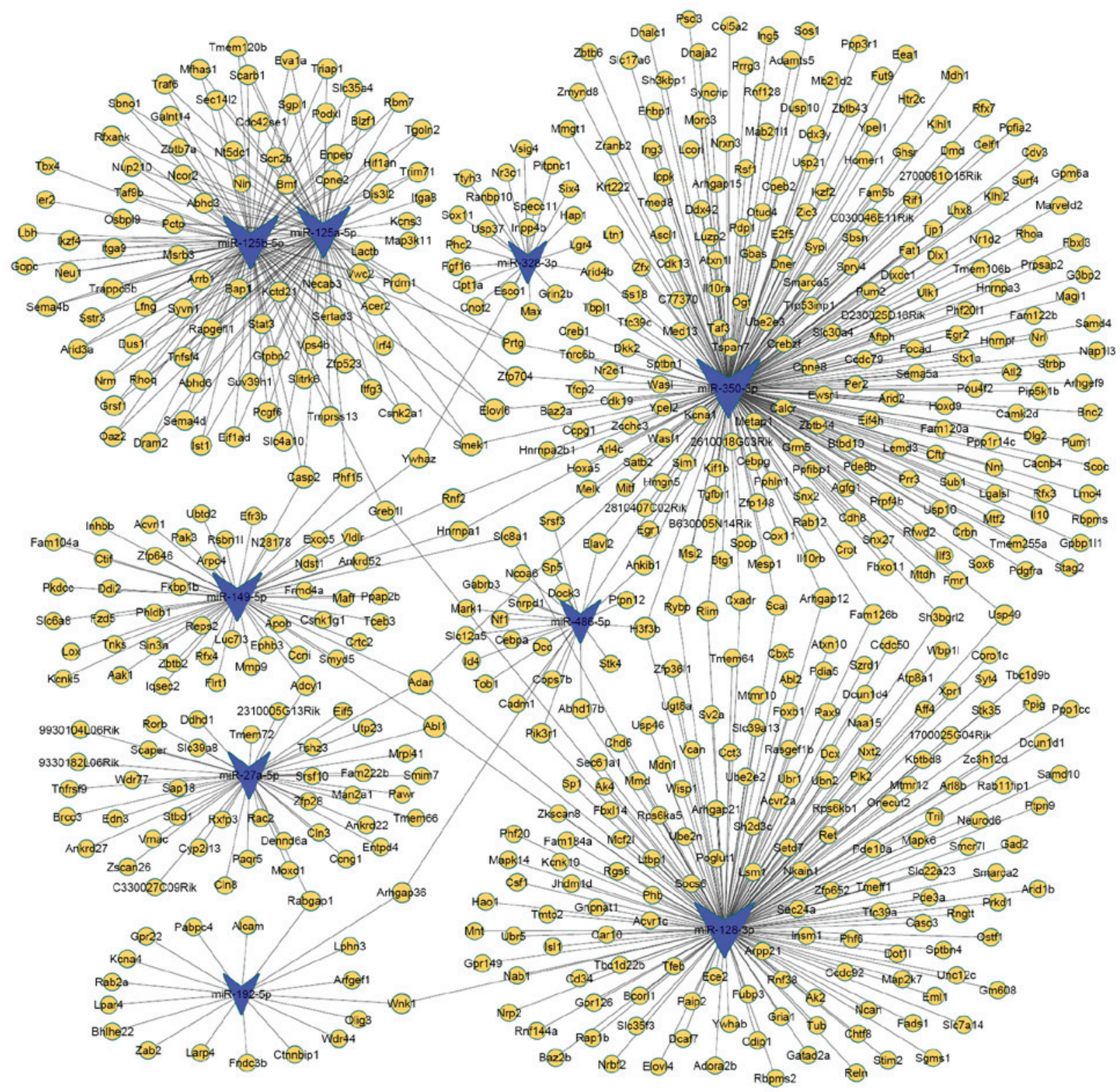

Figure 4. The network of miR-486, miR-192, miR-128, miR-125a, miR-149, miR-27a, miR-125b, miR-350 and miR-328 and their target genes. miR, microRNA. 
A

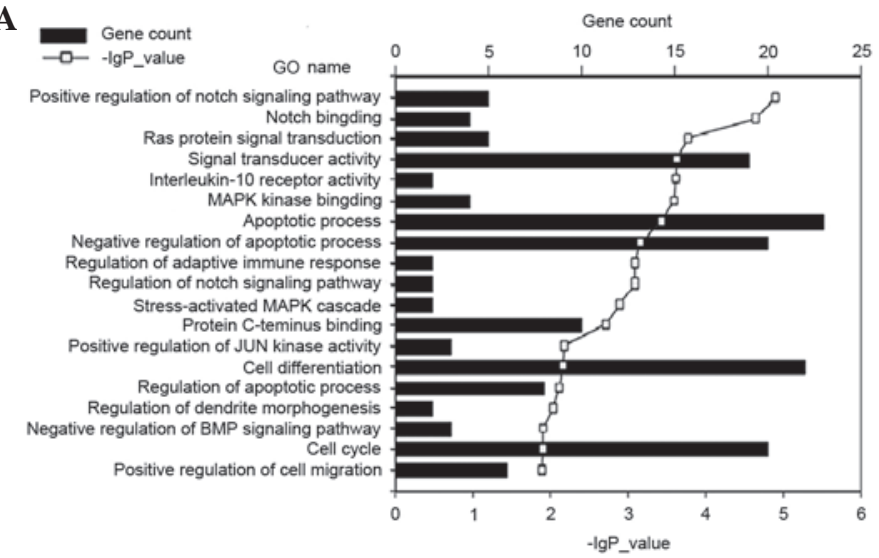

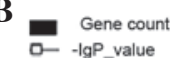

TGF- $\beta$ s signaling pathway MAPK signaling pathway ErbB signaling pathway Ras signaling pathway

T cell recptor signaling pathway TNF signaling pathway Wnt signaling pathway VEGF signaling pathway

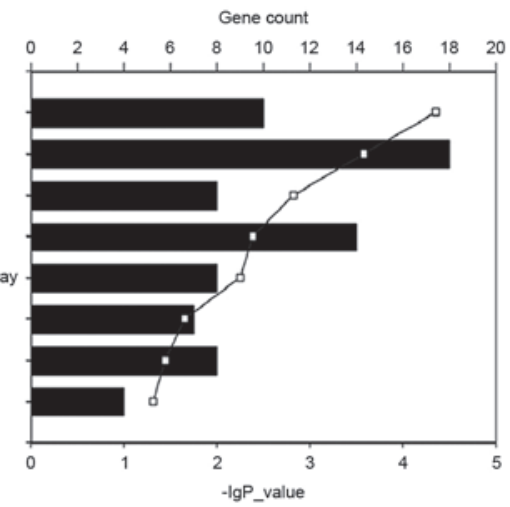

Figure 5. miRNA target prediction and functional annotation analysis. Predicted target genes of the nine reverse transcription-quantitative polymerase chain reaction tested miRNAs were analyzed using (A) GO and (B) KEGG pathway analyses for significant enrichment of genes into functional annotation categories. Significantly enriched categories including cell differentiation, proliferation, apoptotic process, immune response and hematopoiesis are shown with - $\log$ (P-value) and the number of miRNA gene targets. GO, gene ontology; KEGG, Kyoto Encyclopedia of Genes and Genomes; MAPK, mitogen-activated protein kinase; BMP, bone morphogenic protein; TGF- $\beta$, transforming growth factor- $\beta$; TNF, tumor necrosis factor; VEGF, vascular endothelial growth factor.

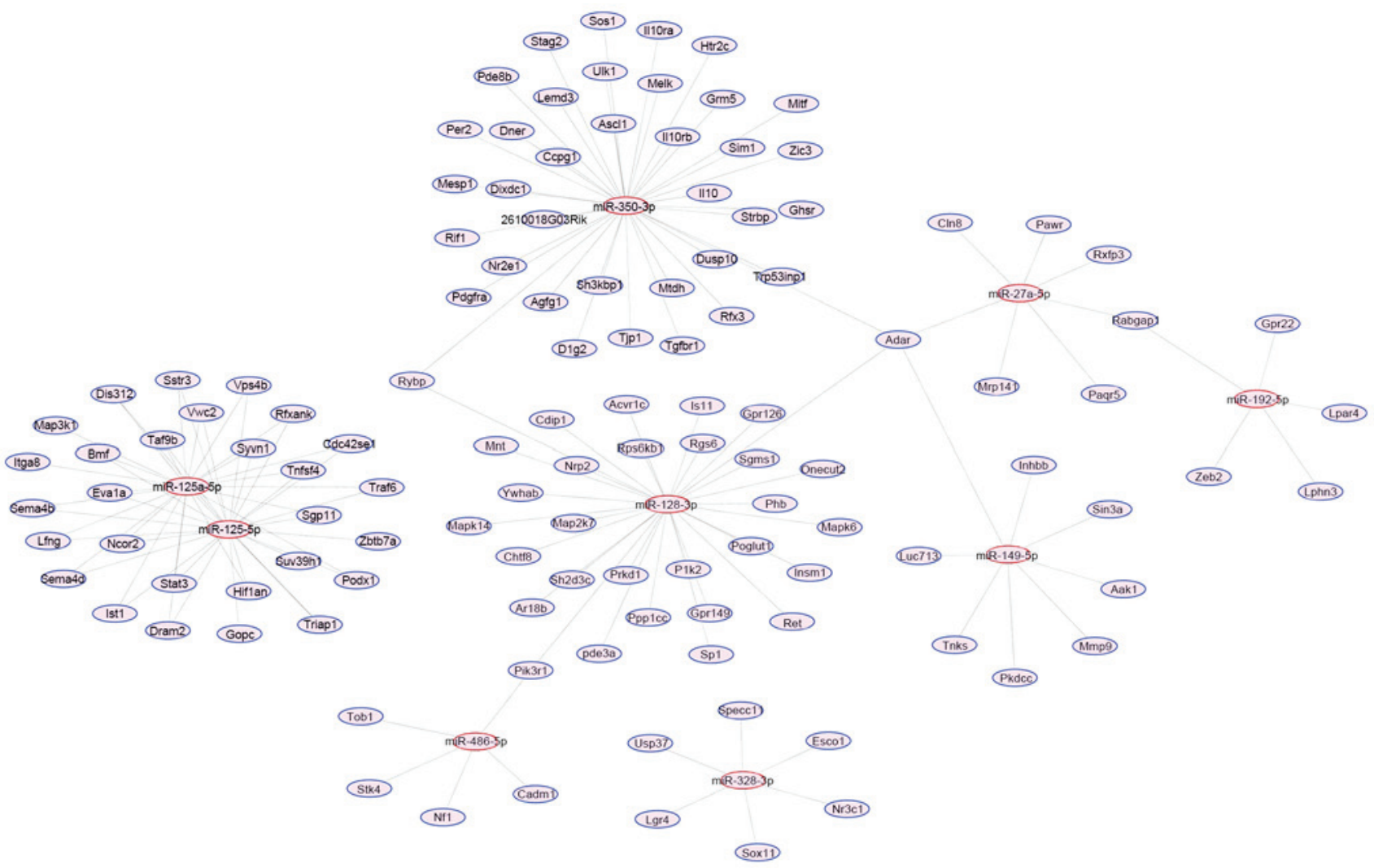

Figure 6. A condensed map of microRNA-target interactions was generated, limited to genes with main function in the regulation of signal transducer activity, apoptotic processes, cell differentiation, cell cycle and the adaptive immune response.

$70-80 \%$ of the whole MDSC population, with M-MDSCs accounting for 20-30\%. G-MDSCs and M-MDSCs have been reported to possess different mechanisms of immunosuppression (18). The present study demonstrated that G-MDSCs accounted for $72.9 \%$ of all MDSCs and $13.5 \%$ of all nucleated cell in the spleens of with transplanted LLCs, which was in accordance with previous reports $(3,18)$.

Previous investigation had revealed that pivotal signaling pathways, for example PI3K, Jak/Stat, Ras and TGF- $\beta$, were required for the development of myeloid cells and producing MDSCs. Targeting these pathways may elucidate the mechanisms that result in expanding MDSCs in cancer (19). Therefore, there is great interest in clarifying the function of signaling pathways that participate in regulate MDSCs. Evidence has demonstrated that miRNAs suppress expression of relevant target genes in hematopoietic cells, and subsequently change the differentiation of hematopoietic progenitor cells. miRNAs may also be involved in the functional regulation of hematopoietic 
stem cells and immune cells (20). MDSCs are immunoregulatory cells originated from hematopoietic stem cells, which may also be regulated by miRNAs. As G-MDSCs have certain different biological characteristics to M-MDSCs, their miRNA expression profiles may also be different. In the current study, the miRNA expression profiles of the G-MDSCs induced by LLC were obtained, which provided a basis to examine how miRNAs are involved in maintaining the characteristics and biological function of G-MDSCs. The data of the current study identified 43 miRNAs with a change of $>1.3$-fold, which were considered as differentially expressed miRNAs between the LLC-induced G-MDSCs and their counterparts from tumor-free mice. The miRNA microarray results from LLC-induced G-MDSCs were different from those of Liu et al (11) using LLC-induced total MDSCs, which agreed with our previous assumption that G-MDSCs and M-MDSCs have different mechanisms of production and function, and therefore different miRNA profiles (11).

In the present study $\mathrm{H} 3 \mathrm{f} 3 \mathrm{~b}$ and Prtg were predicted to be targets of miR-128 and miR-125b, respectively. As reported previously, miR-128 acts as a tumor suppressor, and may inhibit head and neck squamous cell carcinoma growth by targeting H3f3b (21). miR-125 regulates the developmental change in competence of retinal progenitor cells, partly via targeting Prtg (22).

All of these cases indicate that miRNAs are important in the regulation of survival, differentiation and function of LLC-induced G-MDSCs, although the predicted data have not been validated by functional experiments. Further work should be conducted to confirm the effects of these miRNAs in regulating LLC-induced G-MDSCs and the target genes that mediate their functions. In order to translate the findings into clinical use, the data will require validating in MDSCs from patients with cancer.

In summary, to the best of our knowledge, miRNA expression profiles were determined in the G-MDSC subsets from LLC-induced MDSCs for the first time. The data indicated that miRNAs may be important for regulating the survival, differentiation and biological function of tumor-induced G-MDSCs.

\section{Acknowledgements}

This work was supported by the National Natural Science Foundation of China (grant nos. 81101551 and 81302010), Natural Science Foundation of Shanghai (grant no. 13ZR-1405000), the first session Funding Schemes for Young Doctor Training of Shanghai (grant no. 20120443) and the Project to Improve the Ability of Scientific Research for Young Teachers of Fudan University (grant no. 20520133345). We are grateful to Dr Zhang Wu (State Key Laboratory of Medical Genomics and Shanghai Institute of Hematology, Ruijin Hospital, Shanghai Jiao Tong University School of Medicine) for his help in analysis of fluorescence data.

\section{References}

1. Gabrilovich DI and Nagaraj S: Myeloid-derived suppressor cells as regulators of the immune system. Nat Rev Immunol 9: 162-174, 2009 .
2. Condamine T, Ramachandran I, Youn JI and Gabrilovich DI: Regulation of tumor metastasis by myeloid-derived suppressor cells. Annu Rev Med 66: 97-110, 2015

3. Youn JI, Nagaraj S, Collazo M and Gabrilovich DI: Subsets of myeloid-derived suppressor cells in tumor-bearing mice. J Immunol 181: 5791-5802, 2008.

4. Movahedi K, Guilliams M, Van den Bossche J, Van den Bergh R, Gysemans C, Beschin A, De Baetselier P and Van Ginderachter JA: Identification of discrete tumor-induced myeloid-derived suppressor cell subpopulations with distinct T cell-suppressive activity. Blood 111: 4233-4244, 2008.

5. Lim HX, Hong HJ, Cho D and Kim TS: IL-18 enhances immunosuppressive responses by promoting differentiation into monocytic myeloid-derived suppressor cells. J Immunol 193: 5453-5460, 2014.

6. Medina-Echeverz J, Haile LA, Zhao F, Gamrekelashvili J, Ma C, Métais JY, Dunbar CE, Kapoor V, Manns MP, Korangy F and Greten TF: IFN- $\gamma$ regulates survival and function of tumor-induced $\mathrm{CD} 11 \mathrm{~b}^{+} \mathrm{Gr}^{-1}$ high myeloid derived suppressor cells by modulating the anti-apoptotic molecule Bcl2a1. Eur J Immunol 44: 2457-2467, 2014.

7. O'Connell RM, Rao DS, Chaudhuri AA and Baltimore D: Physiological and pathological roles for microRNAs in the immune system. Nat Rev Immunol 10: 111-122, 2010.

8. Ekimler S and Sahin K: Computational methods for MicroRNA target prediction. Genes (Basel) 5: 671-683, 2014.

9. Jiang J, Guo W and Liang X: Phenotypes, accumulation, and functions of myeloid-derived suppressor cells and associated treatment strategies in cancer patients. Hum Immunol 75: 1128-1137, 2014.

10. Zhang M, Liu Q, Mi S, Liang X, Zhang Z, Su X, Liu J, Chen Y, Wang M, Zhang Y, et al: Both miR-17-5p and miR-20a alleviate suppressive potential of myeloid-derived suppressor cells by modulating STAT3 expression. J Immunol 186: 4716-4724, 2011.

11. Liu Y, Lai L, Chen Q, Song Y, Xu S, Ma F, Wang X, Wang J, $\mathrm{Yu} \mathrm{H}$, Cao X and Wang Q: MicroRNA-494 is required for the accumulation and functions of tumor-expanded myeloid-derived suppressor cells via targeting of PTEN. J Immunol 188: 5500-5510, 2012

12. Li L, Zhang J, Diao W, Wang D, Wei Y, Zhang CY and Zen K: MicroRNA-155 and MicroRNA-21 promote the expansion of functional myeloid-derived suppressor cells. J Immunol 192: 1034-1043, 2014.

13. Wang X, Chang X, Zhuo G, Sun M and Yin K: Twist and miR-34a are involved in the generation of tumor-educated myeloid-derived suppressor cells. Int J Mol Sci 14: 20459-20477, 2013.

14. Huang A, Zhang H, Chen S, Xia F, Yang Y, Dong F, Sun D, Xiong $\mathrm{S}$ and Zhang J: miR-34a expands myeloid-derived suppressor cells via apoptosis inhibition. Exp Cell Res 326: 259-266, 2014.

15. Livak KJ and Schmittgen TD: Analysis of relative gene expression data using real-time quantitative PCR and the 2(-Delta Delta C(T)) Method. Methods 25: 402-408, 2001.

16. Cline MS, Smoot M, Cerami E, Kuchinsky A, Landys N, Workman C, Christmas R, Avila-Campilo I, Creech M, Gross B, et al: Integration of biological networks and gene expression data using Cytoscape. Nat Protoc 2: 2366-2382, 2007.

17. Bindea G, Mlecnik B, Hackl H, Charoentong P, Tosolini M, Kirilovsky A, Fridman WH, Pagès F, Trajanoski Z and Galon J: ClueGO: A Cytoscape plug-in to decipher functionally grouped gene ontology and pathway annotation networks. Bioinformatics 25: 1091-1093, 2009.

18. Serafini P: Myeloid derived suppressor cells in physiological and pathological conditions: The good, the bad, and the ugly. Immunol Res 57: 172-184, 2013.

19. Trikha P and Carson WE III: Signaling pathways involved in MDSC regulation. Biochim Biophys Acta 1846: 55-65, 2014.

20. Hukowska-Szematowicz B, Tokarz-Deptula B and Deptula W: MicroRNA (miRNA) and the immune system. Cent Eur J Immunol 37: 387-390, 2012.

21. Hauser B, Zhao Y, Pang X, Ling Z, Myers E, Wang P, Califano J and $\mathrm{Gu}$ X: Functions of miRNA-128 on the regulation of head and neck squamous cell carcinoma growth and apoptosis. PLoS One 10: e0116321, 2015.

22. La Torre A, Georgi S and Reh TA: Conserved microRNA pathway regulates developmental timing of retinal neurogenesis. Proc Natl Acad Sci USA 110: E2362-E2370, 2013. 